\title{
Factors Associated with Anemia Status Among Children Aged 6-59 months in Ghana, 2003-2014
}

\author{
Luke M. Shenton ${ }^{1}$ (1) $\cdot$ Andrew D. Jones ${ }^{2} \cdot$ Mark L. Wilson $^{1}$
}

Published online: 6 February 2020

(c) The Author(s) 2020

\begin{abstract}
Background In 2008, 78\% of Ghanaian children under 5 years old were anemic versus $48 \%$ of children globally. In this study, we identified proximal and distal determinants of severe-moderate anemia and mild anemia related to socioeconomic status, nutrition, and health access.

Methods Using data from the 2003, 2008, and 2014 Ghana Demographic and Health Surveys (GDHS), the odds of severemoderate anemia and mild anemia compared to no anemia, in relation to various hypothesized risk factors, were assessed using a multivariable, multinomial logistic regression, which accounted for survey design.

Results From among 7739 households sampled, severe-moderate anemia was found in approximately $52 \%, 56 \%$, and $40 \%$ of children during 2003, 2008, and 2014, respectively. The proportion of children diagnosed as not anemic was $26 \%$ in 2003, 22\% in 2008, and $33 \%$ in 2014. There are large disparities in anemia prevalence among regions and between urban and rural areas. In 2014, children who were younger (aged 6-24 months), had a recent fever, were from poorer families, and whose mothers were less educated had greater odds of being severely-moderately anemic. These results remained significant when controlling for other risk factors. Predictors of anemia in Ghana remained relatively consistent among the three time periods when the GDHS was administered.

Conclusions The prevalence of anemia in Ghana among children aged 6-59 months has remained unacceptably high. To reduce anemia in these children, the Ghanaian government should concentrate on educating women through both the traditional school system and antenatal care visits.
\end{abstract}

Keywords Anemia disease risk - West Africa - Childhood health disparities · Demographic and health survey (DHS) . Maternal and child nutrition

\section{Significance}

What is Already Known on This Subject? Existing literature using GDHS data shows there are child, household, and sociocultural factors associated with anemia in Ghana.

Luke M. Shenton

1shenton@umich.edu

Andrew D. Jones

jonesand@umich.edu

Mark L. Wilson

wilsonml@umich.edu

1 Department of Epidemiology, School of Public Health, University of Michigan, 1415 Washington Heights, Ann Arbor, MI 48109, USA

2 Department of Nutritional Sciences, School of Public Health, University of Michigan, 1415 Washington Heights, Ann Arbor, MI 48109, USA
What This Study Adds? This is the first article, that we know of, to assess predictors of anemia in Ghana over three rounds of GDHS data. Younger child age, fever in the last 2 weeks, lower household wealth, less maternal education, and maternal anemia were significantly associated with severe-moderate anemia among children in 2014. The predictors remained relatively constant over time, with similar risk factors being seen in 2003 and 2008. These results can inform policy makers in Ghana.

\section{Introduction}

Globally, undernutrition is one of the largest contributors to child mortality, causing an estimated $45 \%$ of total deaths of children younger than five years (Black et al. 2013). Undernutrition encompasses a broad range of public health problems, including deficiencies of micronutrients, especially 
iron (WHO 2008a), that often contribute to anemia. Anemia is of particular concern in children because it can cause deficits in cognitive and behavioral development and function (Beard and Connor 2003; McCann and Ames 2007). Understanding the complex etiologies of various types of anemia is of particular importance, due to the high prevalence of anemia in many parts of the world. One 2011 estimate indicated that $43 \%$ of children under 5 years old had anemia worldwide, with prevalence as high as $71 \%$ in parts of central and western Africa (Stevens et al. 2013).

Because of its large contribution to morbidity and mortality, anemia remains an important public health problem, especially in sub-Saharan Africa. In 2008, 78\% of Ghanaian under-five children were anemic, with $56 \%$ of these children suffering from severe or moderate anemia (Ewusie et al. 2014). Due to this high prevalence, improved understanding of associated factors is needed to better tailor interventions for reducing the burden of anemia in Ghana.

On a biological level, anemia is usually caused by decreased erythrocyte production or increased erythrocyte loss (Camaschella 2015). In addition to such biological causes, there are many complex, upstream environmental, behavioral and social factors that contribute to risk for anemia, especially in children (Balarajan et al. 2011; Siekmans et al. 2014). These factors are often divided into three major categories. First, there are immediate causes, which include inadequate nutrient and absorption (i.e. nutrient supplementation) and exposure to infectious disease (i.e. fever, malaria, intestinal parasites) (Mueller et al. 2017; Paganini and Zimmermann 2017). Secondly, there are underlying causes at the household/family level, such as access to water and sanitation, availability of health services, childcare practice (e.g. breastfeeding) access to diverse food sources, use of insecticide-treated bednets (ITNs), and knowledge about anemia prevention (Larsen et al. 2017). Lastly, the most distal causes are related to the larger sociocultural context, such as education, wealth, and cultural norms and behavior (Ngnie-Teta et al. 2009). Figure 1 provides a graphical depiction of these hypothesized relationships and more information can be found in the methods section. An updated analysis of the relationship between such risk factors and anemia in a nationally representative sample of Ghanaian children is needed given that most existing studies are limited in some way. Investigations into anemia in Ghana generally have involved small sample sizes, and only evaluated risk within small areas (Egbi et al. 2014; Klinkenberg et al. 2006; VanBuskirk et al. 2014). Other studies that used a nationally representative sample are limited by only considering prevalence estimates for anemia or a sample subset of potential predictors (Ewusie et al. 2014; Saaka and Galaa 2017).

The objective of our study, therefore, was to determine the prevalence and predictors of anemia among Ghanaian children aged 6-59 months using nationally representative samples from the Ghana Demographic and Health Survey (GDHS), covering 2003, 2008, and 2014. Thereby, we undertook a more in-depth analyses of anemia risk that also evaluated whether and how there may have been changes in anemia prevalence and correlates over time. We hypothesized many previously identified predictors of childhood anemia, such as malaria and diarrheal disease, would be associated with anemia, as would maternal
Fig. 1 Theoretical framework for predictors of anemia

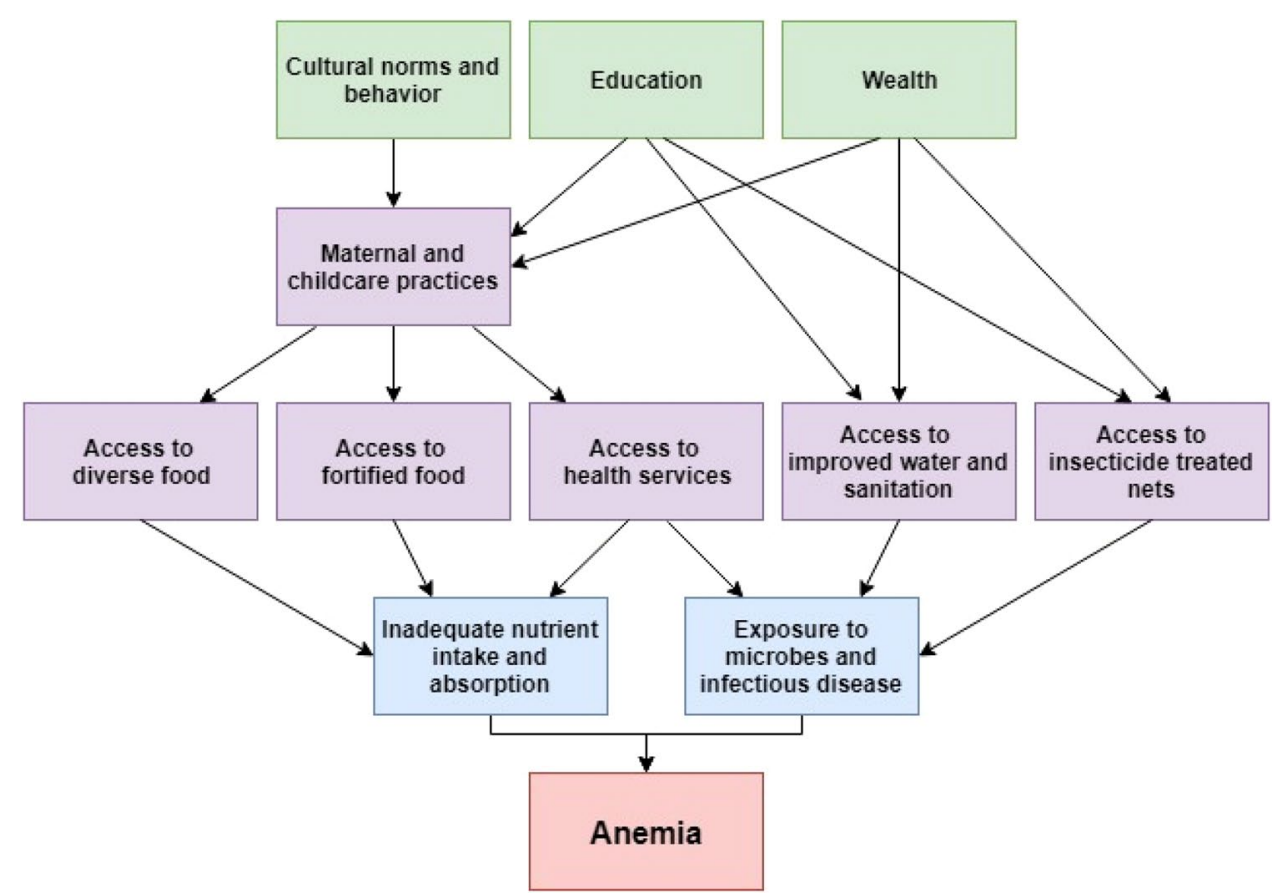


factors including education and maternal autonomy (Balarajan et al. 2011; Siekmans et al. 2014). In addition, we hypothesized there would be decreasing anemia prevalence over time commensurate with improvements in equity across various socioeconomic and demographic groups.

\section{Methods}

\section{Study Population}

This study analyzed Ghana DHS (GDHS) data for 2003, 2008, and 2014. All three surveys and supporting documentation are available from reports published on DHS Program website (Ghana Statistical Service, Ghana Health Service and ICF International 2009, 2015; Ghana Statistical Service, Noguchi Memorial Institute for Medical Research and ORC Macro 2004; United States Agency for International Development 2017). The three surveys used different phases of the DHS; phase IV in 2003, phase $\mathrm{V}$ in 2008, and phase VII in 2014. However, the sampling scheme was virtually identical across these phases, so only the 2014 methods are explained here. The 2014 GDHS used the Ghana 2010 Population and Housing Census as the sampling frame, which consists of 37,641 enumeration areas (EAs) that cover the entire country. The 2008 and 2003 GDHS surveys used the 2000 Population and Housing Census; all regions of Ghana showed population growth between the 2000 and the 2010 census (Ghana Statistical Service 2012). EAs are geographic areas that serve as counting units for the Ghanaian governmental census and contained an average of 145 households in the 2010 census.

The GDHS followed a stratified, two-stage, sample design, which allows estimation of key indicators at the national level, in both urban and rural areas, and in each of Ghana's ten regions. In the first stage, clusters were selected, which consisted of EAs; 216 clusters from urban areas and 211 from rural areas, for a total of 427 clusters.

In the second stage, approximately 30 households were selected from each cluster to yield a total sample size of 12,831 households. The sample design selected an approximately equal sample size in each region; therefore, the sample is not self-weighting at the national level, so weights were calculated such that results are also representative at the national level.

Information about the household, reproductive health, maternal care, childhood immunization, and childcare was collected via in-person interviews with women aged 15-49 years. The population used for analysis was the children from each household aged 6-59 months for whom anemia data are available.

\section{Derived Variables}

Our outcome of interest was anemia status in 6-59 month old children, which we grouped into three levels based on $\mathrm{Hb}$ concentration: severe-moderate anemia $(<100 \mathrm{~g} / \mathrm{L})$, mild anemia (100-109 g/L), or non-anemic ( $\geq 110 \mathrm{~g} / \mathrm{L}$ ) (WHO 2011). We combined severe and moderate anemia into one category because a very small number of children had severe anemia giving the category very low statistical power for analysis. Blood samples were taken via a finger prick (or heel prick for children age 6-11 months) and collected in a microcuvette. A HemoCue analyzer was used to measure $\mathrm{Hb}$ concentration. $\mathrm{Hb}$ levels were adjusted for altitude.

The explanatory variables we analyzed were based on various conceptual models of anemia determinants, plus variables found to be important predictors of anemia in previous studies (Balarajan et al. 2011; Saaka and Galaa 2017; Siekmans et al. 2014). These explanatory variables were related to sociodemographic characteristics and health access, and were examined for any relationship with the children's anemia status.

Immediate causes of anemia, which included recent diarrhea (last 2 weeks), recent fever (last 2 weeks), and recent treatment for parasitic worms (last 6 months), were dichotomized into yes or no answers. Underlying causes comprised most variables, and included age of the child's mother, child's sex, iron supplementation, childcare practice (e.g. breastfeeding), dietary diversity, environmental health (e.g. access to a toilet facility and clean water), access to ITNs, the number of children in the household, and health insurance coverage. Dietary diversity was characterized by the number of different food groups the child consumed in the previous $24 \mathrm{~h}$. We reclassified the GDHS food data into seven distinct food groups: (i) grains, roots and tubers; (ii) legumes and nuts; (iii) dairy products; (iv) flesh foods (meats/fish/poultry); (v) eggs; (vi) vitamin A-rich fruits and vegetables; and (vii) other fruits and vegetables. These food groups underlie the WHO Minimum Dietary Diversity (MDD) indicator for children - an indicator validated against the micronutrient adequacy of child diets (Working Group on Infant and Young Child Feeding Indicators 2006; WHO 2008). The dietary diversity score, which ranged from zero to seven food groups, was then dichotomized to create the MDD that was met by consuming four or more food groups.

The more "distal" hypothesized causes of anemia included household wealth, maternal education, religion, ethnicity, and maternal autonomy. The household wealth index was derived by grouping survey participants into quintiles based on a standardized asset-based score. Measures of maternal autonomy included number of other wives, maternal decision-making autonomy, and maternal attitudes towards being beaten. For decision making autonomy, we classified mothers as having none, some, or full decision-making autonomy 
based on their answers to questions about decision making behaviors in their households. DHS surveyors asked mothers about who in their household makes decisions related to their own health-care, large household purchases, and visits to relatives. Mothers could answer that they alone make the decision, they jointly make the decision with their husband, their husband alone makes the decision, or someone else makes the decisions. If the mother played a role in all decisions she had full decision-making autonomy, if she played a role in making some decisions she had partial decisionmaking autonomy, and if she did not play a role in making decisions she had no decision-making autonomy. For attitudes towards being beaten, we dichotomized mothers' responses to questions about wife beating into yes (beating by their husband is justifiable) or no (not justifiable). DHS surveyors asked mothers if they thought being beaten by their husband was justifiable in five scenarios: they leave the house without telling their husband, they neglect the children, they argue with their husband, they refuse to have sex, and they burn the food.

\section{Statistical Analysis}

The distribution of factors are presented using descriptive statistics, and their unadjusted relationship with severe-moderate and mild anemia were assessed through a Rao-Scott chi-square test. A multivariable, multinomial, logistic regression model was developed to determine the associations between independent factors and anemia status. The regression models were built by considering previous studies, and included all factors other than dietary diversity and breastfeeding. Dietary diversity was not included due to a small overall sample size $(\mathrm{N}=1373)$ and breastfeeding was excluded due to high collinearity with child's age. For all other variables, observations with missing data were removed from the multivariable model. However, the removed observations accounted for only a small proportion of the total number of observations. Ethnicity, originally a nine-category variable, was collapsed into six categories, while maternal education was collapsed from six to three categories, in both cases due to small cell size, which was defined as under 150 observations. The 2003 regression model differed slightly from those of 2008 and 2014 due to missing variables such as iron supplementation, drugs for parasites, and health insurance status. All descriptive, bivariate, and multivariable analyses were conducted using appropriate clustering, stratification, and weighting statements to account for the complex sample design. The regression model generated odds ratios (OR) and $95 \%$ confidence intervals (CI). Variance was estimated through the Taylor series linearization method. Significance was assessed at an $\alpha$ level of 0.05 , with all analyses being done in SAS version 9.4 (SAS Institute, Cary, NC, USA).

\section{Ethical Approval}

This study was exempt from ethical approval because it was limited to publicly available datasets that contained no personally identifiable information. All participants provided informed consent to the GDHS interviewers before being enrolled into the study.

\section{Results}

\section{Study Population}

The 2003, 2008, and 2014 GDHS datasets provided information on 3183,2168 , and 2388 children aged $6-59$ who had $\mathrm{Hb}$ measurements taken, respectively. The sociodemographic characteristics of these children are summarized in Table 1. In 2014, 48\% of children had slept under a mosquito net the previous night; a significant increase from 2003 when only $15 \%$ had. Around $12 \%$ of children had diarrhea in the previous 2 weeks, while $15 \%$ had a fever in the last 2 weeks, an improvement over previous years. A quarter of children in 2014 had no toilet facility compared to one-third in 2003 and $17 \%$ had access to flush toilet in 2014 compared to $7 \%$ in 2003. In 2014, $29 \%$ of mothers (11\% less than in 2003) had completed no education, while only $10 \%$ of mothers had completed secondary education or higher. Approximately 58\% of mothers had full decision-making autonomy in the household, while $7 \%$ had none; in contrast to the $26 \%$ and $40 \%$ of mothers with no and full decision-making autonomy, respectively in 2003. Across all survey years, the majority $(75 \%)$ of children belonged to Christian households and were members of the Akan ethnic group (48\%).

\section{Prevalence and Distribution of Anemia}

Severe-moderate anemia was found in 52\%, 56\%, and $40 \%$ of children during 2003, 2008, and 2014, respectively. The proportion of children diagnosed as not anemic was $26 \%$ in 2003, 22\% in 2008, and 33\% in 2014 (Tables 2, 3, 4). As compared to 2003, the prevalence of severe-moderate anemia increased slightly (4\%) in 2008, but had substantially decreased (12\%) by 2014 . In $2014,48 \%$ of children in rural areas had severe-moderate anemia compared to $30 \%$ in urban areas. While this significant disparity persisted through 2014, both urban and rural areas had a $10 \%$ drop in severe-moderate anemia as compared with 2003. severe-moderate anemia prevalence also differed 
Table 1 Sociodemographic profile of children 6-59 months

\begin{tabular}{|c|c|c|c|c|c|c|}
\hline \multirow[t]{2}{*}{ Characteristics } & \multicolumn{2}{|l|}{2003} & \multicolumn{2}{|l|}{2008} & \multicolumn{2}{|l|}{2014} \\
\hline & $\mathrm{n}$ & $\begin{array}{l}\text { Weighted percent- } \\
\text { ages }(95 \% \text { confidence } \\
\text { intervals) }\end{array}$ & $\mathrm{n}$ & $\begin{array}{l}\text { Weighted percent- } \\
\text { ages }(95 \% \text { confidence } \\
\text { intervals) }\end{array}$ & $\mathrm{n}$ & $\begin{array}{l}\text { Weighted percent- } \\
\text { ages (95\% confidence } \\
\text { intervals) }\end{array}$ \\
\hline Anemia type & 3183 & & 2168 & & 2388 & \\
\hline Severe-moderate & & $52.11(49.95,54.27)$ & & $55.81(52.99,58.63)$ & & $39.80(36.78,42.83)$ \\
\hline Mild & & $22.02(20.39,23.64)$ & & $22.61(20.54,24.67)$ & & $27.00(24.89,29.11)$ \\
\hline None & & $25.87(23.98,27.76)$ & & $21.58(19.34,23.83)$ & & $33.20(30.18,36.22)$ \\
\hline Region & 3183 & & 2168 & & 2388 & \\
\hline Western & & $10.13(8.13,12.12)$ & & $9.43(7.48,11.38)$ & & $10.59(8.69,12.50)$ \\
\hline Central & & $8.86(6.55,11.16)$ & & $9.01(7.01,11.00)$ & & $11.69(7.20,16.18)$ \\
\hline Greater Accra & & $10.73(8.92,12.54)$ & & $11.92(9.83,14.02)$ & & $15.62(12.51,18.73)$ \\
\hline Volta & & $8.51(7.47,9.56)$ & & $8.74(6.81,10.68)$ & & $7.34(5.67,9.00)$ \\
\hline Eastern & & $9.62(8.22,11.03)$ & & $8.62(7.28,9.96)$ & & $8.65(7.10,10.20)$ \\
\hline Ashanti & & $17.71(15.87,19.55)$ & & $19.21(16.60,21.81)$ & & $17.36(14.65,20.06)$ \\
\hline Brong Ahafo & & $11.25(9.93,12.56)$ & & $10.44(8.71,12.17)$ & & $9.60(7.69,11.51)$ \\
\hline Northern & & $13.78(11.97,15.60)$ & & $14.97(12.50,17.43)$ & & $12.52(9.63,15.40)$ \\
\hline Upper east & & $3.09(2.43,3.76)$ & & $4.85(3.73,5.98)$ & & $4.10(2.76,5.44)$ \\
\hline Upper west & & $6.32(5.06,7.58)$ & & $2.81(2.22,3.40)$ & & $2.53(1.85,3.21)$ \\
\hline Urbanicity & 3183 & & 2168 & & 2388 & \\
\hline Urban & & $32.14(29.78,34.50)$ & & $36.95(33.90,40.00)$ & & $45.81(41.73,49.88)$ \\
\hline Rural & & $67.86(65.50,70.22)$ & & $63.05(60.00,66.10)$ & & $54.19(50.12,58.27)$ \\
\hline Child's age (months) & 3183 & & 2168 & & 2388 & \\
\hline $6-11$ & & $21.56(20.30,22.81)$ & & $11.40(9.83,12.97)$ & & $11.28(9.50,13.06)$ \\
\hline $12-23$ & & $21.68(20.00,23.37)$ & & $23.82(22.06,25.59)$ & & $24.41(22.57,26.24)$ \\
\hline $24-35$ & & $19.45(17.94,20.96)$ & & $21.37(19.35,23.39)$ & & $22.59(20.52,24.65)$ \\
\hline $36-47$ & & $20.16(18.74,21.58)$ & & $20.66(18.90,22.42)$ & & $21.48(19.53,23.42)$ \\
\hline $48-59$ & & $17.15(15.81,18.49)$ & & $22.75(20.87,24.62)$ & & $20.25(18.35,22.14)$ \\
\hline Sex & 3183 & & 2168 & & 2388 & \\
\hline Male & & $50.55(48.73,52.36)$ & & $51.45(49.11,53.80)$ & & $52.95(50.59,55.31)$ \\
\hline Female & & $49.45(47.64,51.27)$ & & $48.55(46.20,50.89)$ & & $47.05(44.69,49.41)$ \\
\hline Child slept under net previous night & 3183 & & 2168 & & 2388 & \\
\hline No & & $84.65(82.56,86.73)$ & & $56.05(52.97,59.14)$ & & $52.22(48.84,55.60)$ \\
\hline Yes & & $15.35(13.27,17.44)$ & & $43.95(40.86,47.04)$ & & $47.78(44.40,51.16)$ \\
\hline Had diarrhea recently & 3172 & & 2163 & & 2388 & \\
\hline No & & $84.11(82.63,85.60)$ & & $77.59(75.28,79.81)$ & & $87.39(85.46,89.31)$ \\
\hline Yes, last 2 weeks & & $15.89(14.40,17.37)$ & & $22.41(20.19,24.62)$ & & $12.62(10.69,14.54)$ \\
\hline Had fever recently & 3157 & & 2162 & & 2388 & \\
\hline No & & $77.99(76.31,79.68)$ & & $77.51(75.28,79.74)$ & & $84.72(82.48,86.95)$ \\
\hline Yes, last 2 weeks & & $22.01(20.32,23.69)$ & & $22.49(20.26,24.72)$ & & $15.28(13.05,17.52)$ \\
\hline Iron supplementation & & & 2156 & & 2379 & \\
\hline No & & & & $71.94(69.40,74.49)$ & & $76.77(73.96,79.59)$ \\
\hline Yes & & & & $28.06(25.51,30.60)$ & & $23.23(20.41,26.04)$ \\
\hline Drugs for parasites in last 6 mo & & & 2151 & & 2383 & \\
\hline No & & & & $57.23(54.55,59.91)$ & & $62.17(59.18,65.15)$ \\
\hline Yes & & & & $42.77(40.09,45.45)$ & & $37.83(34.85,40.82)$ \\
\hline Breastfeeding & 3148 & & 2159 & & 2387 & \\
\hline Previously breastfed & & $56.58(55.00,58.16)$ & & $67.40(65.37,69.42)$ & & $68.00(65.89,70.10)$ \\
\hline Never breastfed & & $0.37(0.15,0.58)$ & & $1.57(0.91,2.23)$ & & $1.03(0.52,1.54)$ \\
\hline Still breastfeeding & & $43.06(41.47,44.64)$ & & $31.04(29.06,33.01)$ & & $30.98(28.84,33.11)$ \\
\hline Dietary diversity (no. of food groups) & & & 1684 & & 1373 & \\
\hline
\end{tabular}


Table 1 (continued)

\begin{tabular}{|c|c|c|c|c|c|c|}
\hline \multirow[t]{2}{*}{ Characteristics } & \multicolumn{2}{|l|}{2003} & \multicolumn{2}{|l|}{2008} & \multicolumn{2}{|l|}{2014} \\
\hline & $\mathrm{n}$ & $\begin{array}{l}\text { Weighted percent- } \\
\text { ages ( } 95 \% \text { confidence } \\
\text { intervals) }\end{array}$ & $\mathrm{n}$ & $\begin{array}{l}\text { Weighted percent- } \\
\text { ages ( } 95 \% \text { confidence } \\
\text { intervals) }\end{array}$ & $\mathrm{n}$ & $\begin{array}{l}\text { Weighted percent- } \\
\text { ages }(95 \% \text { confidence } \\
\text { intervals) }\end{array}$ \\
\hline$<4$ & & & & $64.75(61.52,67.98)$ & & $80.65(77.41,83.89)$ \\
\hline$\geq 4$ & & & & $35.25(32.02,38.48)$ & & $19.35(16.11,22.59)$ \\
\hline Water source & 3155 & & 2156 & & 2343 & \\
\hline Piped water & & $28.32(24.85,31.80)$ & & $33.76(29.59,37.93)$ & & $27.72(24.17,31.26)$ \\
\hline Open well water & & $13.85(11.29,16.41)$ & & $36.84(32.69,40.99)$ & & $30.83(26.35,35.31)$ \\
\hline Protected well water & & $31.42(27.75,35.10)$ & & $7.62(5.12,10.12)$ & & $10.10(7.49,12.70)$ \\
\hline Surface water & & $25.63(21.67,29.58)$ & & $15.54(11.89,19.19)$ & & $10.70(7.80,13.61)$ \\
\hline Bottled/sachet water & & $0.78(0.32,1.23)$ & & $6.24(4.46,8.01)$ & & $20.65(17.47,23.84)$ \\
\hline Toilet & 3156 & & & & 2343 & \\
\hline Flush toilet & & $6.72(4.85,8.59)$ & & $8.48(6.57,10.40)$ & & $17.72(14.67,20.77)$ \\
\hline Pit toilet latrine & & $62.05(58.72,65.37)$ & & $62.68(58.75,66.61)$ & & $56.84(52.72,60.95)$ \\
\hline No facility & & $31.23(28.10,34.37)$ & & $28.69(25.13,32.54)$ & & $25.44(21.64,29.25)$ \\
\hline Household wealth quintile & 3183 & & 2168 & & 2388 & \\
\hline Poorest & & $26.50(23.72,29.29)$ & & $26.10(22.61,29.59)$ & & $22.82(19.39,26.25)$ \\
\hline Poorer & & $22.51(20.00,25.01)$ & & $23.35(20.55,26.15)$ & & $20.47(18.02,22.92)$ \\
\hline Middle & & $20.14(17.71,22.58)$ & & $17.63(15.18,20.08)$ & & $20.01(17.18,22.85)$ \\
\hline Richer & & $16.50(14.39,18.60)$ & & $19.02(16.55,21.49)$ & & $18.34(15.24,21.44)$ \\
\hline Richest & & $14.35(12.05,16.65)$ & & $13.90(11.66,16.13)$ & & $18.35(15.09,21.62)$ \\
\hline Mother's age (years) & 3183 & & 2168 & & 2388 & \\
\hline $15-19$ & & $3.54(2.89,4.18)$ & & $3.45(2.56,4.34)$ & & $2.74(1.75,3.73)$ \\
\hline $20-24$ & & $18.14(16.28,20.00)$ & & $18.62(16.46,20.78)$ & & $15.89(13.88,17.89)$ \\
\hline $25-29$ & & $26.38(24.32,28.44)$ & & $27.94(25.31,30.57)$ & & $24.31(21.48,27.15)$ \\
\hline $30-34$ & & $22.18(20.25,24.12)$ & & $20.38(18.20,22.55)$ & & $25.90(23.25,28.54)$ \\
\hline $35-39$ & & $17.35(15.52,19.18)$ & & $18.05(15.98,20.12)$ & & $19.65(17.20,22.11)$ \\
\hline$\geq 40$ & & $12.42(10.91,13.92)$ & & $11.56(9.94,13.18)$ & & $11.21(9.78,13.24)$ \\
\hline Maternal education & 3183 & & 2168 & & 2388 & \\
\hline No education & & $40.30(37.45,43.14)$ & & $33.18(30.08,36.28)$ & & $28.88(25.23,32.53)$ \\
\hline Incomplete primary & & $16.53(14.72,18.34)$ & & $18.34(16.09,20.58)$ & & $13.85(11.74,15.97)$ \\
\hline Complete primary & & $6.64(5.45,7.82)$ & & $6.28(5.01,7.55)$ & & $5.89(4.59,7.19)$ \\
\hline Incomplete secondary & & $32.66(30.44,34.89)$ & & $35.23(32.25,38.20)$ & & $41.15(37.48,44.82)$ \\
\hline Complete secondary & & $2.78(1.90,3.67)$ & & $4.63(3.42,5.85)$ & & $6.99(5.40,8.59)$ \\
\hline Higher & & $1.09(0.59,1.60)$ & & $2.35(1.48,3.22)$ & & $3.24(2.02,4.45)$ \\
\hline Health insurance coverage & & & 2166 & & 2388 & \\
\hline No & & & & $62.41(59.22,65.61)$ & & $33.25(30.18,36.33)$ \\
\hline Yes & & & & $37.59(34.39,40.78)$ & & $66.75(63.67,69.82)$ \\
\hline Maternal anemia type & 3147 & & 2150 & & 2370 & \\
\hline Severe-moderate & & $9.38(8.07,10.69)$ & & $20.28(17.88,22.67)$ & & $9.81(8.18,11.44)$ \\
\hline Mild & & $37.90(35.70,40.10)$ & & $41.31(38.54,44.08)$ & & $34.06(31.33,36.80)$ \\
\hline Not anemic & & $52.72(50.27,55.17)$ & & $38.41(35.37,41.45)$ & & $56.13(53.06,59.19)$ \\
\hline Number of children in household & 3183 & & 2168 & & 2388 & \\
\hline $1-2$ & & $38.72(36.36,41.08)$ & & $40.66(37.65,43.66)$ & & $42.94(40.05,45.82)$ \\
\hline 3 & & $43.95(41.60,46.30)$ & & $41.81(38.53,45.09)$ & & $42.11(39.04,45.18)$ \\
\hline$\geq 4$ & & $17.33(15.25,19.41)$ & & $17.53(14.62,20.45)$ & & $14.95(12.11,17.80)$ \\
\hline Number of other wives & 3182 & & 1965 & & 2094 & \\
\hline 0 & & $71.02(68.71,73.33)$ & & $81.15(78.34,83.95)$ & & $84.96(82.41,87.51)$ \\
\hline 1 & & $28.98(26.67,31.29)$ & & $18.86(16.05,21.66)$ & & $15.04(12.49,17.59)$ \\
\hline Maternal decision-making autonomy & 3134 & & 1965 & & 2108 & \\
\hline
\end{tabular}


Table 1 (continued)

\begin{tabular}{|c|c|c|c|c|c|c|}
\hline \multirow[t]{2}{*}{ Characteristics } & \multicolumn{2}{|l|}{2003} & \multicolumn{2}{|l|}{2008} & \multicolumn{2}{|l|}{2014} \\
\hline & $\mathrm{n}$ & $\begin{array}{l}\text { Weighted percent- } \\
\text { ages }(95 \% \text { confidence } \\
\text { intervals) }\end{array}$ & $\mathrm{n}$ & $\begin{array}{l}\text { Weighted percent- } \\
\text { ages ( } 95 \% \text { confidence } \\
\text { intervals) }\end{array}$ & $\mathrm{n}$ & $\begin{array}{l}\text { Weighted percent- } \\
\text { ages (95\% confidence } \\
\text { intervals) }\end{array}$ \\
\hline No autonomy & & $25.52(22.94,28.10)$ & & $10.27(8.03,12.52)$ & & $7.71(5.61,9.81)$ \\
\hline Partial autonomy & & $34.95(32.20,37.70)$ & & $42.07(38.84,45.30)$ & & $34.70(31.08,38.33)$ \\
\hline Full autonomy & & $39.53(36.66,42.40)$ & & $47.66(43.86,51.45)$ & & $57.59(53.88,61.29)$ \\
\hline Maternal attitudes that justify beating & 3182 & & 2168 & & 2388 & \\
\hline No & & $45.63(42.93,48.33)$ & & $40.98(37.73,44.23)$ & & $66.42(62.48,70.36)$ \\
\hline Yes & & $54.37(51.67,57.07)$ & & $59.02(55.77,62.27)$ & & $33.58(29.64,37.52)$ \\
\hline Religion & 3181 & & 2163 & & 2388 & \\
\hline Christian & & $71.37(68.68,74.06)$ & & $71.29(67.75,74.84)$ & & $74.90(71.13,78.67)$ \\
\hline Islam & & $17.83(15.21,20.45)$ & & $17.77(14.08,21.46)$ & & $18.20(14.37,22.03)$ \\
\hline Traditional & & $4.29(3.15,5.43)$ & & $6.83(4.51,9.14)$ & & $3.39(1.61,5.18)$ \\
\hline No religion & & $6.51(4.94,8.09)$ & & $4.11(2.98,5.24)$ & & $3.51(2.47,4.54)$ \\
\hline Ethnicity & 3181 & & 2166 & & 2388 & \\
\hline Akan & & $46.27(42.98,49.56)$ & & $47.10(43.31,50.89)$ & & $48.07(43.30,52.84)$ \\
\hline $\mathrm{Ga} /$ Dangme & & $7.13(5.35,8.91)$ & & $4.59(3.04,6.15)$ & & $5.67(3.89,7.44)$ \\
\hline Ewe & & $12.67(10.89,14.45)$ & & $12.43(9.92,14.94)$ & & $13.08(10.51,15.64)$ \\
\hline Mole-Dagbani & & $16.67(14.12,19.21)$ & & $20.19(16.64,23.73)$ & & $17.02(1337,20.68)$ \\
\hline Gurma & & $4.36(2.54,6.18)$ & & $5.49(3.33,7.65)$ & & $8.31(5.22,11.39)$ \\
\hline Other & & $12.90(10.62,15.19)$ & & $10.20(7.94,12.47)$ & & $7.85(5.94,9.77)$ \\
\hline
\end{tabular}

Ghana Demographic and Health Survey, 2003-2014

by province across all survey years, ranging from $29 \%$ in Ashanti region (2014) to $62 \%$ in the Northern region (2014).

\section{Factors Associated with Mild Anemia}

In the 2014 multivariable logistic regression model, few factors were significantly associated with mild anemia (Table 5). Children aged 48-59 months were less likely to be anemic (OR $0.49 ; 95 \%$ CI 0.31, 0.79) than children aged 12-23 months. Children of mothers with severe-moderate anemia were more likely to be anemic (OR 2.04, 95\% CI 1.13, 3.66) than children whose mothers did not have severe-moderate anemia. In 2008, no factors examined were significantly associated with mild anemia. In 2003, child's age (OR $0.43,95 \%$ CI $0.30,0.64$ ) and mother's anemia status (OR 1.65, 95\% CI 1.11, 2.45) were associated with child's anemia status; in addition, using open well water versus piped water was associated with higher odds of mild anemia (OR 1.62, 95\% CI 1.03, 2.55).

\section{Factors Associated with Severe-Moderate Anemia}

In 2014, child's age had a strong association with severe-moderate anemia. As children aged, their odds of anemia became progressively lower; as compared to children 12-23 months old, those aged 24-35 months had 0.48 (95\% CI $0.32,0.73)$ times the odds of anemia, and others aged $48-59$ months had 0.20 (95\% CI 0.13, 0.31) times the odds of anemia. Fever in the previous 2 weeks was significantly associated with increased odds of severe-moderate anemia (OR 2.39, 95\% CI 1.58, 3.63).

The other variables significantly associated with childhood severe-moderate anemia in 2014 were directly related to maternal characteristics or household factors. Mothers who had secondary or higher education were less likely to have moderately to severely anemic children compared to uneducated mothers (OR 0.53, 95\% CI 0.34, 0.81). Children were significantly more likely to be moderately-severely anemic when their mothers had mild anemia (OR 1.80, 95\% CI $1.28,2.54$ ) or severe-moderate anemia (OR $2.66,95 \%$ CI $1.46,4.83)$. Lastly, children in the richest wealth quintile had 
Table 2 Rao-Scott chi-square bivariate analysis of anemia type by sociodemographic factors for children

6-59 months from the 2014 Ghana Demographic and Health Survey $(\mathrm{n}=2388)$

\begin{tabular}{|c|c|c|c|c|}
\hline & $\begin{array}{l}\text { Severe-moderate } \\
\text { anemia (weighted \%) }\end{array}$ & $\begin{array}{l}\text { Mild anemia } \\
\text { (weighted \%) }\end{array}$ & $\begin{array}{l}\text { Non-anemic } \\
\text { (weighted \%) }\end{array}$ & P-value \\
\hline Region & & & & $<0.0001$ \\
\hline Western & 37.94 & 29.78 & 32.28 & \\
\hline Central & 46.70 & 26.66 & 26.65 & \\
\hline Greater Accra & 29.55 & 28.81 & 41.64 & \\
\hline Volta & 43.45 & 27.25 & 29.30 & \\
\hline Eastern & 38.52 & 28.00 & 33.48 & \\
\hline Ashanti & 29.32 & 24.79 & 45.89 & \\
\hline Brong Ahafo & 34.02 & 29.52 & 36.46 & \\
\hline Northern & 61.57 & 22.93 & 15.50 & \\
\hline Upper east & 43.95 & 30.61 & 25.44 & \\
\hline Upper west & 52.28 & 21.36 & 26.36 & \\
\hline Urbanicity & & & & $<0.0001$ \\
\hline Urban & 30.48 & 28.99 & 40.54 & \\
\hline Rural & 47.69 & 25.31 & 27.00 & \\
\hline Child's age (months) & & & & $<0.0001$ \\
\hline $6-11$ & 52.16 & 26.26 & 21.58 & \\
\hline $12-23$ & 52.25 & 24.61 & 23.14 & \\
\hline $24-35$ & 35.20 & 30.86 & 33.94 & \\
\hline $36-47$ & 33.77 & 27.44 & 38.79 & \\
\hline $48-59$ & 29.45 & 25.50 & 45.05 & \\
\hline Sex & & & & 0.8514 \\
\hline Male & 39.21 & 27.50 & 33.30 & \\
\hline Female & 40.47 & 26.44 & 33.09 & \\
\hline Child slept under net previous night & & & & 0.0404 \\
\hline No & 36.70 & 27.97 & 35.33 & \\
\hline Yes & 43.20 & 25.93 & 30.87 & \\
\hline Had diarrhea recently & & & & 0.0158 \\
\hline No & 38.48 & 27.40 & 34.12 & \\
\hline Yes, last 2 weeks & 49.00 & 24.19 & 26.81 & \\
\hline Had fever recently & & & & $<0.0001$ \\
\hline No & 36.85 & 27.75 & 35.40 & \\
\hline Yes, last 2 weeks & 56.15 & 22.84 & 21.02 & \\
\hline Iron supplementation & & & & 0.0015 \\
\hline No & 42.09 & 25.23 & 32.67 & \\
\hline Yes & 34.75 & 32.91 & 34.75 & \\
\hline Drugs for parasites in last $6 \mathrm{mo}$ & & & & $<0.0001$ \\
\hline No & 44.10 & 26.18 & 29.73 & \\
\hline Yes & 32.69 & 28.36 & 38.95 & \\
\hline Breastfeeding & & & & $<0.0001$ \\
\hline Ever breastfed & 32.97 & 28.38 & 38.65 & \\
\hline Never breastfed & 43.89 & 17.84 & 38.26 & \\
\hline Still breastfeeding & 54.74 & 24.14 & 21.11 & \\
\hline Dietary diversity (no. of food groups) & & & & 0.2195 \\
\hline$<4$ & 48.75 & 25.11 & 26.14 & \\
\hline$\geq 4$ & 40.72 & 28.44 & 30.84 & \\
\hline Water source & & & & $<0.0001$ \\
\hline Piped water & 35.44 & 27.07 & 37.49 & \\
\hline Tube well water & 49.98 & 23.38 & 26.64 & \\
\hline Dug well & 50.21 & 23.85 & 25.94 & \\
\hline Surface water & 52.08 & 25.79 & 22.13 & \\
\hline
\end{tabular}


Table 2 (continued)

\begin{tabular}{|c|c|c|c|c|}
\hline & $\begin{array}{l}\text { Severe-moderate } \\
\text { anemia (weighted \%) }\end{array}$ & $\begin{array}{l}\text { Mild anemia } \\
\text { (weighted \%) }\end{array}$ & $\begin{array}{l}\text { Non-anemic } \\
\text { (weighted \%) }\end{array}$ & P-value \\
\hline Bottled/sachet water & 20.74 & 32.79 & 46.46 & \\
\hline Toilet & & & & $<0.0001$ \\
\hline Flush toilet & 18.36 & 34.19 & 47.46 & \\
\hline Pit toilet latrine & 40.32 & 24.84 & 34.84 & \\
\hline No facility & 54.98 & 25.45 & 19.57 & \\
\hline Wealth & & & & $<0.0001$ \\
\hline Poorest & 54.96 & 25.81 & 19.23 & \\
\hline Poorer & 51.02 & 25.60 & 23.38 & \\
\hline Middle & 43.21 & 20.11 & 36.69 & \\
\hline Richer & 26.47 & 33.64 & 39.89 & \\
\hline Richest & 18.05 & 30.91 & 51.04 & \\
\hline Mother's age (years) & & & & 0.1247 \\
\hline $15-19$ & 59.09 & 18.41 & 22.50 & \\
\hline $20-24$ & 46.00 & 26.88 & 27.12 & \\
\hline $25-29$ & 39.63 & 26.09 & 34.28 & \\
\hline $30-34$ & 36.21 & 27.14 & 36.64 & \\
\hline $35-39$ & 37.78 & 27.84 & 34.38 & \\
\hline$\geq 40$ & 38.54 & 29.35 & 32.11 & \\
\hline Maternal education & & & & $<0.0001$ \\
\hline No education & 54.20 & 25.93 & 19.87 & \\
\hline Incomplete primary & 41.57 & 27.42 & 31.01 & \\
\hline Complete primary & 48.48 & 18.32 & 33.20 & \\
\hline Incomplete secondary & 32.74 & 27.81 & 39.45 & \\
\hline Complete secondary & 24.54 & 30.31 & 45.15 & \\
\hline Higher & 10.76 & 33.03 & 56.21 & \\
\hline Covered by health insurance & & & & 0.0006 \\
\hline No & 46.57 & 25.80 & 27.63 & \\
\hline Yes & 36.43 & 27.59 & 35.97 & \\
\hline Mother's anemia level & & & & $<0.0001$ \\
\hline Moderate-severe & 53.76 & 27.69 & 18.55 & \\
\hline Mild & 45.40 & 25.59 & 29.00 & \\
\hline Not anemic & 34.06 & 27.82 & 38.12 & \\
\hline Number of children in household & & & & $<0.0001$ \\
\hline $1-2$ & 32.58 & 28.39 & 39.03 & \\
\hline 3 & 44.15 & 25.66 & 30.19 & \\
\hline$\geq 4$ & 48.31 & 26.77 & 24.92 & \\
\hline Number of other wives & & & & $<0.0001$ \\
\hline 0 & 37.60 & 26.51 & 35.89 & \\
\hline$\geq 1$ & 53.05 & 27.26 & 37.60 & \\
\hline Maternal decision-making autonomy & & & & 0.0336 \\
\hline No autonomy & 51.50 & 25.86 & 22.64 & \\
\hline Partial autonomy & 41.57 & 27.17 & 31.27 & \\
\hline Full autonomy & 37.24 & 26.79 & 35.97 & \\
\hline Maternal attitudes that justify beating & & & & $<0.0001$ \\
\hline No & 34.27 & 23.75 & 25.51 & \\
\hline Yes & 50.75 & 28.64 & 37.09 & \\
\hline Religion & & & & $<0.0001$ \\
\hline Christian & 36.23 & 27.21 & 36.56 & \\
\hline Islam & 46.92 & 27.04 & 26.04 & \\
\hline Traditional & 72.76 & 15.85 & 11.39 & \\
\hline
\end{tabular}


Table 2 (continued)

\begin{tabular}{lllll}
\hline & $\begin{array}{l}\text { Severe-moderate } \\
\text { anemia (weighted \%) }\end{array}$ & $\begin{array}{l}\text { Mild anemia } \\
(\text { weighted \%) }\end{array}$ & $\begin{array}{l}\text { Non-anemic } \\
\text { (weighted \%) }\end{array}$ & P-value \\
\hline $\begin{array}{l}\text { No religion } \\
\text { Ethnicity }\end{array}$ & 47.22 & 33.00 & 19.78 & $<0.0001$ \\
Akan & & & & \\
Ga/Dangme & 34.76 & 25.83 & 39.41 & \\
Ewe & 37.51 & 29.30 & 33.20 & \\
Mole-Dagbani & 36.15 & 30.29 & 33.56 & \\
Gurma & 45.83 & 26.50 & 27.67 & \\
Other & 59.39 & 26.15 & 14.46 & \\
\hline
\end{tabular}

P-values show whether there is a statistically significant difference between categories of each variable

0.33 (95\% CI $0.14,0.74)$ times the odds of severe-moderate anemia compared to children in the poorest wealth quintile (Table 5).

In 2008, child's age, fever in the previous 2 weeks, and maternal anemia status were significantly associated with childhood severe-moderate anemia, similar to 2014. However, household wealth (OR 0.92, 95\% CI 0.57, 1.49) and maternal education (OR $0.58,95 \%$ CI $0.24,1.40$ ) were not associated with childhood severe-moderate anemia. In 2008, unlike in 2014, children were significantly less likely to have severe-moderate anemia if their mother's decision-making autonomy was partial (OR 0.53, 95\% CI: $0.31,0.90$ ) or full (OR 0.56, 95\% CI 0.32, 0.98). Furthermore, those children in families with health insurance had lower odds of anemia compared to other children without access to health insurance (OR 0.69, 95\% CI 0.51, 0.93).

In 2003, child's age was significantly associated with severe-moderate anemia, similar to 2008 and 2014. As in 2014, but unlike 2008, household wealth and maternal education did have a significant association with severe-moderate anemia during childhood in 2003.

\section{Discussion}

Anemia prevalence in Ghana is exceptionally high. Globally, anemia prevalence in young children (6-59 months old) is estimated at $43 \%$ as compared with $67 \%$ in Ghana (Stevens et al. 2013). From our analysis, 40\% of Ghanaian children aged 6-59 months had severe-moderate anemia in 2014. Although this represents a substantial improvement over 2003 and 2008, there is evidence of widening disparities across regions of Ghana. In 2003, severe-moderate anemia prevalence difference was $23 \%$ between the highest (Northern region) and the lowest (Greater Accra region). However, that gap increased to $34 \%$ in 2008 and $32 \%$ in 2014. There was also an urban-rural disparity in severe-moderate anemia prevalence (17\% higher in rural areas in 2014), but this disparity has remained relatively constant over time.

Across all three survey years, child's age was negatively associated with severe-moderate anemia, as older children had lower odds of anemia than younger children. The high prevalence of severe-moderate anemia in children under 2 years old appears related to poor maternal nutrition, as children born to malnourished mothers have poor stores of many essential micronutrients, such as iron, zinc, vitamin $A$ and $B_{12}$ and folate (Kotecha 2011; Neumann et al. 2004). Our finding that children with mothers who have anemia have higher odds of developing anemia themselves, provides further evidence for this (Table 5).

Complementary foods and feeding practices are especially important for determining the micronutrient adequacy of 6-23 month-old children, as breast milk makes a progressively smaller contribution to an infant's nutritional requirements into late infancy and early childhood (Saaka and Galaa 2017; Woldie et al. 2015). Our findings are consistent with this observation, in that the prevalence of severe-moderate anemia was higher among children who were still being breastfed than those who never or formerly breastfed (Tables 2, 3, 4). This likely reflects inadequate consumption of high quality complementary foods with continued breastfeeding rather than a deleterious effect of continued breastfeeding itself on iron status or anemia (Cumber et al. 2017; Lander et al. 2009). Reverse causality may also underlie this association (i.e., smaller, malnourished children may 
Table 3 Rao-Scott chi-square bivariate analysis of anemia type by sociodemographic factors for children

6-59 months from the 2008 Ghana Demographic and Health Survey $(\mathrm{n}=2168)$

\begin{tabular}{|c|c|c|c|c|}
\hline & $\begin{array}{l}\text { Severe-moderate } \\
\text { anemia (weighted \%) }\end{array}$ & $\begin{array}{l}\text { Mild anemia } \\
\text { (weighted \%) }\end{array}$ & $\begin{array}{l}\text { Non anemic } \\
\text { (weighted \%) }\end{array}$ & P-value \\
\hline Region & & & & $<0.0001$ \\
\hline Western & 62.03 & 17.36 & 20.61 & \\
\hline Central & 62.55 & 22.89 & 14.57 & \\
\hline Greater Accra & 30.99 & 31.33 & 37.68 & \\
\hline Volta & 60.26 & 19.21 & 20.53 & \\
\hline Eastern & 48.28 & 25.11 & 26.61 & \\
\hline Ashanti & 56.38 & 23.39 & 20.23 & \\
\hline Brong Ahafo & 54.12 & 23.91 & 21.98 & \\
\hline Northern & 65.47 & 16.54 & 17.99 & \\
\hline Upper east & 58.33 & 30.62 & 11.05 & \\
\hline Upper west & 74.51 & 13.55 & 11.94 & \\
\hline Urbanicity & & & & $<0.0001$ \\
\hline Urban & 43.29 & 24.29 & 32.41 & \\
\hline Rural & 63.15 & 21.62 & 15.23 & \\
\hline Child's age (months) & & & & $<0.0001$ \\
\hline $6-11$ & 67.34 & 17.89 & 14.77 & \\
\hline $12-23$ & 64.33 & 20.76 & 14.90 & \\
\hline $24-35$ & 57.61 & 22.40 & 19.98 & \\
\hline $36-47$ & 48.47 & 25.57 & 25.96 & \\
\hline $48-59$ & 46.08 & 24.41 & 29.52 & \\
\hline Sex & & & & 0.4260 \\
\hline Male & 56.77 & 22.86 & 20.37 & \\
\hline Female & 54.80 & 22.34 & 22.86 & \\
\hline Child slept under net previous night & & & & 0.1338 \\
\hline No & 54.02 & 22.78 & 23.19 & \\
\hline Yes & 58.09 & 22.38 & 19.53 & \\
\hline Had diarrhea recently & & & & $<0.0001$ \\
\hline No & 53.12 & 23.16 & 23.72 & \\
\hline Yes, last 2 weeks & 65.26 & 20.55 & 14.19 & \\
\hline Had fever recently & & & & 0.0015 \\
\hline No & 53.80 & 22.84 & 23.36 & \\
\hline Yes, last 2 weeks & 62.49 & 22.00 & 15.51 & \\
\hline Iron supplementation & & & & 0.6630 \\
\hline No & 55.80 & 23.08 & 21.12 & \\
\hline Yes & 55.70 & 21.48 & 22.82 & \\
\hline Drugs for parasites in last $6 \mathrm{mo}$ & & & & 0.0021 \\
\hline No & 58.91 & 22.32 & 18.77 & \\
\hline Yes & 51.83 & 23.02 & 25.15 & \\
\hline Breastfeeding & & & & $<0.0001$ \\
\hline Ever breastfed & 50.70 & 23.98 & 25.32 & \\
\hline Never breastfed & 40.41 & 24.99 & 34.60 & \\
\hline Still breastfeeding & 67.44 & 19.82 & 12.74 & \\
\hline Dietary diversity (no. of food groups) & & & & 0.9970 \\
\hline$<4$ & 59.61 & 21.46 & 18.92 & \\
\hline$\geq 4$ & 59.50 & 21.40 & 19.10 & \\
\hline Water source & & & & $<0.0001$ \\
\hline Piped water & 48.52 & 24.99 & 26.49 & \\
\hline Tube well water & 62.51 & 22.21 & 15.28 & \\
\hline Dug well & 54.07 & 24.49 & 21.44 & \\
\hline Surface water & 66.68 & 17.05 & 16.27 & \\
\hline
\end{tabular}


Table 3 (continued)

\begin{tabular}{|c|c|c|c|c|}
\hline & $\begin{array}{l}\text { Severe-moderate } \\
\text { anemia (weighted \%) }\end{array}$ & $\begin{array}{l}\text { Mild anemia } \\
\text { (weighted \%) }\end{array}$ & $\begin{array}{l}\text { Non anemic } \\
\text { (weighted \%) }\end{array}$ & $\mathrm{P}$-value \\
\hline Bottled/sachet water & 30.01 & 23.87 & 46.11 & \\
\hline Toilet & & & & $<0.0001$ \\
\hline Flush toilet & 28.56 & 28.91 & 42.52 & \\
\hline Pit toilet latrine & 54.53 & 23.52 & 21.94 & \\
\hline No facility & 66.38 & 18.74 & 14.88 & \\
\hline Wealth & & & & $<0.0001$ \\
\hline Poorest & 66.84 & 20.35 & 12.81 & \\
\hline Poorer & 61.55 & 22.28 & 16.17 & \\
\hline Middle & 60.53 & 20.33 & 19.14 & \\
\hline Richer & 48.57 & 21.85 & 29.57 & \\
\hline Richest & 29.36 & 31.31 & 39.32 & \\
\hline Mother's age (years) & & & & 0.0198 \\
\hline $15-19$ & 68.88 & 19.98 & 11.14 & \\
\hline $20-24$ & 59.76 & 23.77 & 16.46 & \\
\hline $25-29$ & 57.89 & 21.11 & 21.01 & \\
\hline $30-34$ & 48.43 & 25.65 & 25.93 & \\
\hline $35-39$ & 54.61 & 22.35 & 23.04 & \\
\hline$\geq 40$ & 55.42 & 20.18 & 24.41 & \\
\hline Maternal education & & & & $<0.0001$ \\
\hline No education & 64.95 & 18.45 & 16.60 & \\
\hline Incomplete primary & 58.58 & 22.56 & 18.87 & \\
\hline Complete primary & 53.48 & 26.02 & 20.50 & \\
\hline Incomplete secondary & 50.85 & 25.40 & 23.75 & \\
\hline Complete secondary & 36.16 & 23.24 & 40.60 & \\
\hline Higher & 24.52 & 29.40 & 46.08 & \\
\hline Covered by health insurance & & & & $<0.0001$ \\
\hline No & 59.79 & 21.39 & 18.82 & \\
\hline Yes & 49.12 & 24.67 & 26.21 & \\
\hline Mother's anemia level & & & & $<0.0001$ \\
\hline Moderate-severe & 69.23 & 17.80 & 12.97 & \\
\hline Mild & 56.79 & 22.41 & 20.80 & \\
\hline Not anemic & 47.44 & 25.48 & 27.08 & \\
\hline Number of children in household & & & & 0.1567 \\
\hline $1-2$ & 53.88 & 22.85 & 23.28 & \\
\hline 3 & 55.17 & 23.01 & 21.82 & \\
\hline$\geq 4$ & 61.82 & 21.10 & 17.08 & \\
\hline Number of other wives & & & & 0.0023 \\
\hline 0 & 54.23 & 22.86 & 22.91 & \\
\hline$\geq 1$ & 64.73 & 19.40 & 15.87 & \\
\hline Maternal decision-making autonomy & & & & 0.0489 \\
\hline No autonomy & 65.03 & 20.87 & 14.10 & \\
\hline Partial autonomy & 56.70 & 21.19 & 22.11 & \\
\hline Full autonomy & 53.89 & 23.68 & 22.43 & \\
\hline Maternal attitudes that justify beating & & & & $<0.0001$ \\
\hline No & 50.71 & 25.29 & 24.00 & \\
\hline Yes & 63.16 & 18.74 & 18.10 & \\
\hline Religion & & & & 0.0008 \\
\hline Christian & 52.88 & 23.78 & 23.33 & \\
\hline Islam & 62.28 & 17.92 & 19.81 & \\
\hline Traditional & 66.75 & 20.64 & 12.61 & \\
\hline
\end{tabular}


Table 3 (continued)

\begin{tabular}{lllll}
\hline & $\begin{array}{l}\text { Severe-moderate } \\
\text { anemia (weighted \%) }\end{array}$ & $\begin{array}{l}\text { Mild anemia } \\
(\text { weighted \%) }\end{array}$ & $\begin{array}{l}\text { Non anemic } \\
(\text { weighted \%) }\end{array}$ & P-value \\
\hline $\begin{array}{l}\text { No religion } \\
\text { Ethnicity }\end{array}$ & 61.40 & 24.93 & 13.68 & 0.0010 \\
Akan & & & & \\
Ga/Dangme & 52.56 & 23.61 & 23.83 & \\
Ewe & 43.75 & 26.47 & 29.78 & \\
Mole-Dagbani & 55.67 & 24.10 & 20.23 \\
Gurma & 60.98 & 19.96 & 19.07 & \\
Other & 74.56 & 17.16 & 8.27 \\
\hline
\end{tabular}

$\mathrm{P}$-values show whether there is a statistically significant difference between categories of each variable

be weaned later because of perceived vulnerability) (Habicht 2000; Simondon et al. 2001).

Infants also generally have a higher incidence of infectious diseases, which can reduce their ability to ingest and absorb iron, perhaps further explaining the higher prevalence of anemia among younger children (Villalpando et al. 2003). From bivariate analyses across all years, we determined that a higher prevalence of anemia was associated with diarrhea or fever experienced within the previous 2 weeks (Tables 2 , $3,4)$. Although diarrhea and fever were associated with anemia, the cross-sectional nature of observations precludes determining the direction of the association. Participantreported diarrhea or fever was asked for a period preceding the $\mathrm{Hb}$ measurement, although we cannot determine the temporal pattern of any association. One published prospective study has suggested that anemia may be a precursor of diarrheal disease (Levy et al. 2005). The relationship could also be cyclic, with diarrhea initially decreasing the body's ability to ingest and absorb iron, hence increasing subsequent risk of anemia, and anemia then increasing the risk of additional diarrheal episodes (Semba et al. 2008).

The association between fever in the past 2 weeks and severe-moderate anemia persisted in the multivariable model for all survey years, however, this was not the case for diarrheal illness. In our model, we controlled for both water source and toilet type (often a proxy for sanitation), which could have attenuated any association between diarrheal illness and anemia, as cleaner water and improved sanitation are associated with a lower risk of diarrheal pathogen exposure and illness (WHO 2014). More broadly, socioeconomic status (e.g. household wealth and education) also has been shown to predict health outcomes (Cameron and Williams
2009; Feinstein 1993). We included both household wealth and education in our multivariable models, which could have controlled for the association between two health indicators, such as diarrhea and anemia (see Fig. 1).

In 2003 and 2014, children from higher-income households and children with more highly educated mothers had lower odds of severe-moderate anemia in multivariable analysis. (For 2008, these associations were only significant in bivariate analysis.) It seems reasonable that households with higher incomes are better equipped to keep their children healthy due to a greater ability to purchase health services and food with higher nutritional value. Additionally, highly educated mothers are more likely to be knowledgeable about how to care for their own health, and that of their child.

\section{Limitations and Strengths}

Our findings should be interpreted within the context of several limitations. The cross-sectional nature of the GDHS allows us to look for statistical associations, but not to assess temporality or causality. Survivor bias is also a possible issue; we only included surviving children and deaths could have resulted from complications due to anemia. Although this study analyzed data from three rounds of the GDHS, and each year's survey was similar, some variables such as health insurance coverage, iron supplementation, and parasites treatment were only available in later datasets, requiring slightly different multivariable models by year. Additionally, all datasets lacked information on factors such as malaria infection, so we were unable to consider the impact they had on anemia. 
Table 4 Rao-Scott chi-square bivariate analysis of anemia type by sociodemographic factors for children

6-59 months from the 2003 Ghana Demographic and Health Survey $(\mathrm{n}=3183)$

\begin{tabular}{|c|c|c|c|c|}
\hline & $\begin{array}{l}\text { Severe-moderate } \\
\text { anemia (weighted \%) }\end{array}$ & $\begin{array}{l}\text { Mild anemia } \\
\text { (weighted \%) }\end{array}$ & $\begin{array}{l}\text { Non anemic } \\
\text { (weighted \%) }\end{array}$ & P-value \\
\hline Region & & & & $<0.0001$ \\
\hline Western & 56.33 & 22.33 & 21.34 & \\
\hline Central & 52.54 & 23.85 & 23.60 & \\
\hline Greater Accra & 37.33 & 23.32 & 39.35 & \\
\hline Volta & 48.52 & 24.76 & 26.71 & \\
\hline Eastern & 48.26 & 20.67 & 21.07 & \\
\hline Ashanti & 55.34 & 21.91 & 22.75 & \\
\hline Brong Ahafo & 50.44 & 21.39 & 28.18 & \\
\hline Northern & 60.12 & 19.01 & 20.87 & \\
\hline Upper east & 51.50 & 22.49 & 26.01 & \\
\hline Upper west & 57.30 & 22.83 & 19.87 & \\
\hline Urbanicity & & & & $<0.0001$ \\
\hline Urban & 40.94 & 25.27 & 33.79 & \\
\hline Rural & 57.40 & 20.47 & 22.12 & \\
\hline Child's age (months) & & & & $<0.0001$ \\
\hline $6-11$ & 47.39 & 18.52 & 34.09 & \\
\hline $12-23$ & 64.60 & 19.12 & 16.28 & \\
\hline $24-35$ & 55.75 & 21.59 & 22.65 & \\
\hline $36-47$ & 49.91 & 24.17 & 25.92 & \\
\hline $48-59$ & 40.72 & 28.01 & 31.27 & \\
\hline Sex & & & & 0.9986 \\
\hline Male & 52.06 & 22.04 & 25.90 & \\
\hline Female & 52.16 & 21.99 & 25.84 & \\
\hline Child slept under net previous night & & & & 0.3841 \\
\hline No & 52.64 & 21.90 & 25.47 & \\
\hline Yes & 49.21 & 22.68 & 28.11 & \\
\hline Had diarrhea recently & & & & 0.0043 \\
\hline No & 50.85 & 22.09 & 27.06 & \\
\hline Yes, last 2 weeks & 58.66 & 21.46 & 19.88 & \\
\hline Had fever recently & & & & $<0.0001$ \\
\hline No & 49.58 & 22.60 & 27.82 & \\
\hline Yes, last 2 weeks & 60.56 & 20.49 & 18.95 & \\
\hline \multicolumn{5}{|l|}{ Iron supplementation } \\
\hline \multicolumn{5}{|l|}{ No } \\
\hline \multicolumn{5}{|l|}{ Yes } \\
\hline \multicolumn{5}{|l|}{ Drugs for parasites in last 6 mo } \\
\hline \multicolumn{5}{|l|}{ No } \\
\hline \multicolumn{5}{|l|}{ Yes } \\
\hline Breastfeeding & & & & 0.0002 \\
\hline Ever breastfed & 48.53 & 24.69 & 26.78 & \\
\hline Never breastfed & 34.67 & 26.55 & 38.78 & \\
\hline Still breastfeeding & 57.16 & 18.54 & 24.29 & \\
\hline \multicolumn{5}{|l|}{ Dietary diversity (no. of food groups) } \\
\hline \multicolumn{5}{|l|}{$<4$} \\
\hline \multicolumn{5}{|l|}{$\geq 4$} \\
\hline Water source & & & & $<0.0001$ \\
\hline Piped water & 41.10 & 24.24 & 34.66 & \\
\hline Open well water & 56.11 & 24.73 & 19.17 & \\
\hline Protected well water & 56.78 & 19.90 & 23.31 & \\
\hline Surface water & 56.77 & 20.21 & 23.02 & \\
\hline
\end{tabular}


Table 4 (continued)

\begin{tabular}{|c|c|c|c|c|}
\hline & $\begin{array}{l}\text { Severe-moderate } \\
\text { anemia (weighted \%) }\end{array}$ & $\begin{array}{l}\text { Mild anemia } \\
\text { (weighted \%) }\end{array}$ & $\begin{array}{l}\text { Non anemic } \\
\text { (weighted \%) }\end{array}$ & P-value \\
\hline Bottled/sachet water & 45.98 & 30.72 & 23.30 & \\
\hline Toilet & & & & $<0.0001$ \\
\hline Flush toilet & 30.92 & 32.23 & 36.84 & \\
\hline Pit toilet latrine & 51.36 & 21.70 & 26.94 & \\
\hline No facility & 58.02 & 20.42 & 21.56 & \\
\hline Wealth & & & & $<0.0001$ \\
\hline Poorest & 61.72 & 18.77 & 19.50 & \\
\hline Poorer & 56.41 & 21.85 & 21.73 & \\
\hline Middle & 54.38 & 22.50 & 23.13 & \\
\hline Richer & 44.76 & 22.13 & 33.11 & \\
\hline Richest & 32.89 & 27.45 & 39.66 & \\
\hline Mother's age (years) & & & & 0.0689 \\
\hline $15-19$ & 49.48 & 25.07 & 25.45 & \\
\hline $20-24$ & 58.67 & 17.09 & 24.23 & \\
\hline $25-29$ & 52.53 & 22.53 & 24.93 & \\
\hline $30-34$ & 47.66 & 25.69 & 25.65 & \\
\hline $35-39$ & 51.66 & 21.74 & 26.60 & \\
\hline$\geq 40$ & 50.97 & 21.06 & 27.97 & \\
\hline Maternal education & & & & $<0.0001$ \\
\hline No education & 58.74 & 20.20 & 21.05 & \\
\hline Incomplete primary & 54.06 & 22.07 & 23.87 & \\
\hline Complete primary & 54.49 & 24.28 & 21.23 & \\
\hline Incomplete secondary & 44.53 & 23.34 & 32.13 & \\
\hline Complete secondary & 33.29 & 29.19 & 37.52 & \\
\hline Higher & 38.34 & 16.58 & 45.08 & \\
\hline \multicolumn{5}{|l|}{ Covered by health insurance } \\
\hline \multicolumn{5}{|l|}{ No } \\
\hline \multicolumn{5}{|l|}{ Yes } \\
\hline Mother's anemia level & & & & 0.0013 \\
\hline Moderate-severe & 48.65 & 22.80 & 28.55 & \\
\hline Mild & 55.99 & 20.63 & 23.38 & \\
\hline Not anemic & 56.33 & 23.82 & 19.85 & \\
\hline Number of children in household & & & & 0.0030 \\
\hline $1-2$ & 47.59 & 23.71 & 28.71 & \\
\hline 3 & 53.93 & 21.77 & 24.31 & \\
\hline$\geq 4$ & 57.62 & 18.87 & 23.51 & \\
\hline Number of other wives & & & & $<0.0001$ \\
\hline 0 & 49.67 & 22.25 & 28.08 & \\
\hline$\geq 1$ & 58.23 & 21.51 & 20.26 & \\
\hline Maternal decision-making autonomy & & & & 0.0093 \\
\hline No autonomy & 54.43 & 21.77 & 23.80 & \\
\hline Partial autonomy & 54.58 & 18.97 & 26.46 & \\
\hline Full autonomy & 48.60 & 24.93 & 26.46 & \\
\hline Maternal attitudes that justify beating & & & & 0.0033 \\
\hline No & 54.58 & 22.15 & 23.27 & \\
\hline Yes & 49.21 & 21.87 & 28.91 & \\
\hline Religion & & & & 0.0547 \\
\hline Christian & 50.52 & 22.16 & 27.32 & \\
\hline Islam & 54.79 & 22.15 & 23.06 & \\
\hline Traditional & 61.99 & 20.40 & 17.61 & \\
\hline
\end{tabular}


Table 4 (continued)

\begin{tabular}{lllll}
\hline & $\begin{array}{l}\text { Severe-moderate } \\
\text { anemia (weighted \%) }\end{array}$ & $\begin{array}{l}\text { Mild anemia } \\
(\text { weighted \%) }\end{array}$ & $\begin{array}{l}\text { Non anemic } \\
(\text { weighted \%) }\end{array}$ & P-value \\
\hline $\begin{array}{l}\text { No religion } \\
\text { Ethnicity }\end{array}$ & 55.85 & 20.69 & 23.47 & 0.0381 \\
Akan & & & & \\
Ga/Dangme & 50.82 & 22.35 & 26.83 & \\
Ewe & 47.49 & 21.38 & 31.12 \\
Mole-Dagbani & 48.96 & 22.35 & 28.70 \\
Gurma & 56.66 & 22.65 & 20.69 \\
Other & 62.98 & 15.94 & 21.08 \\
\hline
\end{tabular}

$\mathrm{P}$-values show whether there is a statistically significant difference between categories of each variabl

This study also has many strengths. The large sample size helped avoid small cell sizes in the multivariable analysis, allowing us to test multiple associations between potential predictors and anemia status with adequate statistical power. To the authors knowledge, this is the first study to assess predictors of anemia in Ghana over three different rounds of GDHS data.

\section{Conclusions}

The prevalence of anemia among children aged 6-59 months in 2014 remains unacceptably high, even though substantial improvement has occurred since 2003. Despite overall advances, Ghana has experienced widening disparities in the prevalence of anemia among regions, and a persistent disparity between urban and rural areas. Results demonstrate that younger child age, fever in the last 2 weeks, lower household wealth, less maternal education, and maternal anemia are significantly associated with greater severe-moderate anemia. Associations have remained relatively consistent over the 2003, 2008 and 2014 time periods. In order to decrease anemia prevalence among children, the Ghanaian government should concentrate on educating women, both through the traditional education system and through antenatal care visits. Antenatal care visits could also provide an opportunity to ensure mothers are receiving adequate nutrition during pregnancy, as this has significant implications for the amount of iron available to infants through breastfeeding. A focus on improving water sources and sanitation in Ghana would also be beneficial, since they are often seen as predictive of diarrheal disease, which may have a cyclic effect on anemia. 
Table 5 Adjusted odds ratios for anemia type by sociodemographic covariates for children 6-59 months from the 2003, 2008, and 2014 Ghana Demographic and Health Surveys

\begin{tabular}{|c|c|c|c|c|c|c|}
\hline \multirow[t]{2}{*}{ Covariates } & \multicolumn{2}{|l|}{$2003(n=3028)$} & \multicolumn{2}{|l|}{$2008(n=1888)$} & \multicolumn{2}{|l|}{$2014(\mathrm{n}=2030)$} \\
\hline & $\begin{array}{l}\text { Severe-moderate } \\
\text { anemia versus non- } \\
\text { anemic }\end{array}$ & $\begin{array}{l}\text { Mild anemia } \\
\text { versus non- } \\
\text { anemic }\end{array}$ & $\begin{array}{l}\text { Severe-moderate } \\
\text { anemia versus non- } \\
\text { anemic }\end{array}$ & $\begin{array}{l}\text { Mild anemia ver- } \\
\text { sus non-anemic }\end{array}$ & $\begin{array}{l}\text { Severe-moderate } \\
\text { anemia versus non- } \\
\text { anemic }\end{array}$ & $\begin{array}{l}\text { Mild anemia ver- } \\
\text { sus non-anemic }\end{array}$ \\
\hline \multicolumn{7}{|l|}{ Urbanicity } \\
\hline Urban & Ref & Ref & Ref & Ref & Ref & Ref \\
\hline Rural & $1.12(0.76,1.66)$ & $0.96(0.62,1.48)$ & $1.61(1.05,2.47)$ & $1.37(0.88,2.13)$ & $0.84(0.55,1.27)$ & $0.92(0.60,1.41)$ \\
\hline \multicolumn{7}{|l|}{$\begin{array}{c}\text { Child's age } \\
\text { (months) }\end{array}$} \\
\hline $6-11$ & $0.31(0.22,0.42)$ & $0.43(0.30,0.64)$ & $0.90(0.52,1.58)$ & $0.84(0.44,1.61)$ & $1.39(0.81,2.38)$ & $1.43(0.82,2.49)$ \\
\hline $12-23$ & Ref & Ref & Ref & Ref & Ref & Ref \\
\hline $24-35$ & $0.61(0.44,0.87)$ & $0.83(0.57,1.21)$ & $0.62(0.39,1.00)$ & $0.71(0.41,1.21)$ & $0.48(0.32,0.73)$ & $1.06(0.69,1.63)$ \\
\hline $36-47$ & $0.46(0.33,0.66)$ & $0.83(0.55,1.23)$ & $0.40(0.25,0.64)$ & $0.67(0.39,1.16)$ & $0.32(0.21,0.48)$ & $0.64(0.42,0.98)$ \\
\hline $48-59$ & $0.32(0.22,0.45)$ & $0.80(0.56,1.14)$ & $0.34(0.22,0.55)$ & $0.54(0.32,0.93)$ & $0.20(0.13,0.31)$ & $0.49(0.31,0.79)$ \\
\hline \multicolumn{7}{|l|}{ Sex } \\
\hline Male & Ref & Ref & Ref & Ref & Ref & Ref \\
\hline Female & $1.07(0.86,1.32)$ & $1.07(0.84,1.36)$ & $0.84(0.65,1.08)$ & $0.81(0.60,1.11)$ & $0.84(0.62,1.14)$ & $0.86(0.61,1.21)$ \\
\hline \multicolumn{7}{|l|}{$\begin{array}{l}\text { Child slept under } \\
\text { net previous night }\end{array}$} \\
\hline No & Ref & Ref & Ref & Ref & Ref & Ref \\
\hline Yes & $0.79(0.59,1.06)$ & $0.96(0.70,1.31)$ & $1.11(0.83,1.50)$ & $1.01(0.72,1.42)$ & $1.03(0.75,1.42)$ & $0.94(0.70,1.28)$ \\
\hline \multicolumn{7}{|l|}{$\begin{array}{l}\text { Had diarrhea } \\
\text { recently }\end{array}$} \\
\hline No & Ref & Ref & Ref & Ref & Ref & Ref \\
\hline Yes, last 2 weeks & $1.14(0.83,1.55)$ & $1.15(0.79,1.67)$ & $1.37(0.93,2.02)$ & $1.37(0.87,2.15)$ & $1.13(0.68,1.85)$ & $1.01(0.60,1.70)$ \\
\hline \multicolumn{7}{|l|}{ Had fever recently } \\
\hline No & Ref & Ref & Ref & Ref & Ref & Ref \\
\hline Yes, last 2 weeks & $1.60(1.20,2.13)$ & $1.20(0.88,1.63)$ & $1.59(1.10,2.29)$ & $1.36(0.88,2.09)$ & $2.39(1.58,3.63)$ & $1.32(0.87,2.01)$ \\
\hline \multicolumn{7}{|l|}{$\begin{array}{l}\text { Iron supplementa- } \\
\text { tion }\end{array}$} \\
\hline No & & & Ref & Ref & Ref & Ref \\
\hline Yes & & & $0.94(0.67,1.33)$ & $0.86(0.57,1.30)$ & $0.76(0.53,1.08)$ & $1.30(0.89,1.88)$ \\
\hline \multicolumn{7}{|l|}{$\begin{array}{l}\text { Drugs for parasites } \\
\text { in last } 6 \text { mo }\end{array}$} \\
\hline No & & & Ref & Ref & Ref & Ref \\
\hline Yes & & & $1.01(0.72,1.42)$ & $1.01(0.71,1.43)$ & $1.05(0.73,1.49)$ & $1.11(0.79,1.55)$ \\
\hline \multicolumn{7}{|l|}{ Water source } \\
\hline Piped water & Ref & Ref & Ref & Ref & Ref & Ref \\
\hline Open well water & $1.34(0.87,2.06)$ & $1.62(1.03,2.55)$ & $1.13(0.71,1.80)$ & $1.28(0.77,2.15)$ & $1.25(0.78,2.01)$ & $1.08(0.69,1.69)$ \\
\hline $\begin{array}{l}\text { Protected well } \\
\text { water }\end{array}$ & $1.10(0.73,1.64)$ & $1.08(0.68,1.71)$ & $1.12(0.58,2.17)$ & $1.60(0.88,2.89)$ & $1.31(0.67,2.55)$ & $1.08(0.58,2.01)$ \\
\hline Surface water & $1.07(0.68,1.68)$ & $1.12(0.67,1.88)$ & $0.99(0.56,1.76)$ & $0.84(0.43,1.64)$ & $1.19(0.66,2.13)$ & $1.23(0.64,2.35)$ \\
\hline $\begin{array}{l}\text { Bottled/sachet } \\
\text { water }\end{array}$ & $2.30(0.58,9.12)$ & $2.10(0.53,8.31)$ & $0.59(0.31,1.14)$ & $0.50(0.23,1.10)$ & $0.91(0.54,1.55)$ & $1.41(0.90,2.21)$ \\
\hline \multicolumn{7}{|l|}{ Toilet } \\
\hline Flush toilet & Ref & Ref & Ref & Ref & Ref & Ref \\
\hline Pit toilet latrine & $1.07(0.67,1.72)$ & $0.69(0.48,0.99)$ & $1.47(0.81,2.67)$ & $1.30(0.71,2.40)$ & $1.51(0.84,2.70)$ & $0.75(0.46,1.21)$ \\
\hline No facility & $1.01(0.60,1.71)$ & $0.69(0.45,1.07)$ & $1.73(0.82,3.63)$ & $1.12(0.52,2.45)$ & $1.93(0.99,3.77)$ & $1.10(0.62,1.95)$ \\
\hline \multicolumn{7}{|l|}{ Wealth } \\
\hline Poorest & Ref & Ref & Ref & Ref & Ref & Ref \\
\hline Poorer & $0.96(0.65,1.40)$ & $1.24(0.85,1.81)$ & $1.13(0.69,1.87)$ & $0.98(0.55,1.75)$ & $1.14(0.72,1.81)$ & $1.11(0.65,1.89)$ \\
\hline
\end{tabular}


Table 5 (continued)

\begin{tabular}{|c|c|c|c|c|c|c|}
\hline \multirow[t]{2}{*}{ Covariates } & \multicolumn{2}{|l|}{$2003(n=3028)$} & \multicolumn{2}{|l|}{$2008(n=1888)$} & \multicolumn{2}{|l|}{$2014(\mathrm{n}=2030)$} \\
\hline & $\begin{array}{l}\text { Severe-moderate } \\
\text { anemia versus non- } \\
\text { anemic }\end{array}$ & $\begin{array}{l}\text { Mild anemia } \\
\text { versus non- } \\
\text { anemic }\end{array}$ & $\begin{array}{l}\text { Severe-moderate } \\
\text { anemia versus non- } \\
\text { anemic }\end{array}$ & $\begin{array}{l}\text { Mild anemia ver- } \\
\text { sus non-anemic }\end{array}$ & $\begin{array}{l}\text { Severe-moderate } \\
\text { anemia versus non- } \\
\text { anemic }\end{array}$ & $\begin{array}{l}\text { Mild anemia ver- } \\
\text { sus non-anemic }\end{array}$ \\
\hline Middle & $0.91(0.60,1.39)$ & $1.27(0.82,1.99)$ & $1.05(0.55,2.01)$ & $0.67(0.32,1.41)$ & $0.67(0.38,1.18)$ & $0.52(0.28,0.96)$ \\
\hline Richer & $0.59(0.34,1.02)$ & $0.82(0.45,1.48)$ & $0.70(0.34,1.43)$ & $0.63(0.30,1.34)$ & $0.48(0.22,1.05)$ & $0.90(0.43,1.89)$ \\
\hline Richest & $0.42(0.22,0.81)$ & $0.87(0.46,1.65)$ & $0.58(0.24,1.40)$ & $1.04(0.41,2.65)$ & $0.33(0.14,0.74)$ & $0.45(0.19,1.09)$ \\
\hline \multicolumn{7}{|l|}{$\begin{array}{l}\text { Mother's age } \\
\text { (years) }\end{array}$} \\
\hline $15-19$ & $0.69(0.38,1.26)$ & $1.14(0.57,2.30)$ & $1.05(0.35,3.22)$ & $1.14(0.31,4.17)$ & $4.95(1.17,21.13)$ & $3.18(0.71,14.22)$ \\
\hline $20-24$ & Ref & Ref & Ref & Ref & Ref & Ref \\
\hline $25-29$ & $0.90(0.65,1.26)$ & $1.30(0.91,1.87)$ & $0.94(0.60,1.48)$ & $0.78(0.46,1.32)$ & $0.86(0.50,1.47)$ & $0.77(0.41,1.42)$ \\
\hline $30-34$ & $0.79(0.57,1.09)$ & $1.33(0.92,1.92)$ & $0.76(0.48,1.22)$ & $0.77(0.43,1.40)$ & $1.03(0.63,1.69)$ & $0.99(0.57,1.72)$ \\
\hline $35-39$ & $0.68(0.48,0.98)$ & $0.97(0.64,1.45)$ & $0.95(0.54,1.66)$ & $0.76(0.39,1.49)$ & $1.08(0.62,1.87)$ & $0.97(0.56,1.70)$ \\
\hline$\geq 40$ & $0.70(0.47,1.05)$ & $0.86(0.53,1.39)$ & $0.75(0.45,1.26)$ & $0.72(0.37,1.38)$ & $0.70(0.40,1.22)$ & $0.89(0.47,1.71)$ \\
\hline \multicolumn{7}{|l|}{ Maternal education } \\
\hline No education & Ref & Ref & Ref & Ref & Ref & Ref \\
\hline $\begin{array}{l}\text { Primary (complete } \\
\text { or incomplete) }\end{array}$ & $0.87(0.63,1.22)$ & $1.03(0.74,1.44)$ & $0.87(0.56,1.36)$ & $1.30(0.81,2.09)$ & $0.67(0.44,1.03)$ & $0.74(0.47,1.16)$ \\
\hline $\begin{array}{l}\text { Secondary or } \\
\text { higher }\end{array}$ & $0.58(0.42,0.79)$ & $0.80(0.57,1.11)$ & $0.92(0.57,1.49)$ & $1.36(0.80,2.32)$ & $0.53(0.34,0.81)$ & $0.81(0.51,1.27)$ \\
\hline \multicolumn{7}{|l|}{$\begin{array}{l}\text { Covered by health } \\
\text { insurance }\end{array}$} \\
\hline No & & & Ref & Ref & Ref & Ref \\
\hline Yes & & & $0.69(0.51,0.93)$ & $0.82(0.57,1.17)$ & $0.74(0.53,1.04)$ & $0.97(0.70,1.36)$ \\
\hline \multicolumn{7}{|l|}{$\begin{array}{l}\text { Mother's Anemia } \\
\text { Level }\end{array}$} \\
\hline Moderate-severe & $1.73(1.19,2.51)$ & $1.65(1.11,2.45)$ & $2.89(1.90,4.38)$ & $1.30(0.81,2.10)$ & $2.66(1.46,4.83)$ & $2.04(1.13,3.66)$ \\
\hline Mild & $1.35(1.10,1.66)$ & $1.11(0.85,1.44)$ & $1.345(0.972,1.86)$ & $1.15(0.78,1.69)$ & $1.80(1.28,2.54)$ & $1.28(0.87,1.89)$ \\
\hline Not anemic & Ref & Ref & Ref & Ref & Ref & Ref \\
\hline \multicolumn{7}{|l|}{$\begin{array}{l}\text { Number of children } \\
\text { in household }\end{array}$} \\
\hline $1-2$ & Ref & Ref & Ref & Ref & Ref & Ref \\
\hline 3 & $1.26(1.02,1.57)$ & $1.02(0.80,1.30)$ & $0.99(0.73,1.34)$ & $1.07(0.75,1.528)$ & $1.32(0.93,1.87)$ & $1.24(0.87,1.76)$ \\
\hline$\geq 4$ & $1.21(0.89,1.64)$ & $0.87(0.62,1.22)$ & $1.04(0.68,1.59)$ & $1.05(0.65,1.689)$ & $1.57(0.97,2.56)$ & $1.51(0.94,2.44)$ \\
\hline \multicolumn{7}{|l|}{$\begin{array}{l}\text { Number of other } \\
\text { wives }\end{array}$} \\
\hline 0 & Ref & Ref & Ref & Ref & Ref & Ref \\
\hline$\geq 1$ & $1.36(1.07,1.71)$ & $1.36(1.05,1.76)$ & $1.13(0.75,1.70)$ & $1.10(0.69,1.748)$ & $1.22(0.78,1.91)$ & $1.21(0.73,2.00)$ \\
\hline \multicolumn{7}{|l|}{$\begin{array}{l}\text { Maternal decision- } \\
\text { making autonomy }\end{array}$} \\
\hline No autonomy & Ref & Ref & Ref & Ref & Ref & Ref \\
\hline Partial autonomy & $0.94(0.67,1.25)$ & $0.78(0.56,1.07)$ & $0.53(0.31,0.90)$ & $0.60(0.35 .1 .008)$ & $1.02(0.50,2.05)$ & $0.84(0.42,1.68)$ \\
\hline Full autonomy & $0.90(0.67,1.22)$ & $1.06(0.77,1.45)$ & $0.56(0.32,0.98)$ & $0.67(0.38,1.206)$ & $0.69(0.36,1.32)$ & $0.81(0.41,1.60)$ \\
\hline \multicolumn{7}{|l|}{$\begin{array}{l}\text { Maternal attitudes } \\
\text { that justify beat- } \\
\text { ing }\end{array}$} \\
\hline Yes & Ref & Ref & Ref & Ref & Ref & Ref \\
\hline No & $0.97(0.77,1.22)$ & $0.90(0.70,1.16)$ & $1.00(0.73,1.37)$ & $1.41(1.00,2.00)$ & $0.81(0.58,1.12)$ & $1.01(0.74,1.36)$ \\
\hline \multicolumn{7}{|l|}{ Religion } \\
\hline Christian & Ref & Ref & Ref & Ref & Ref & Ref \\
\hline Islam & $1.03(0.74,1.42)$ & $1.02(0.70,1.49)$ & $1.14(0.67,1.93)$ & $0.78(0.42,1.450)$ & $1.35(0.81,2.24)$ & $1.19(0.75,1.86)$ \\
\hline Traditional & $1.17(0.74,1.86)$ & $1.28(0.71,2.29)$ & $1.14(0.61,2.13)$ & $1.19(0.56,2.503)$ & $2.02(0.74,5.50)$ & $1.01(0.31,3.22)$ \\
\hline
\end{tabular}


Table 5 (continued)

\begin{tabular}{|c|c|c|c|c|c|c|}
\hline \multirow[t]{2}{*}{ Covariates } & \multicolumn{2}{|l|}{$2003(n=3028)$} & \multicolumn{2}{|l|}{$2008(n=1888)$} & \multicolumn{2}{|l|}{$2014(n=2030)$} \\
\hline & $\begin{array}{l}\text { Severe-moderate } \\
\text { anemia versus non- } \\
\text { anemic }\end{array}$ & $\begin{array}{l}\text { Mild anemia } \\
\text { versus non- } \\
\text { anemic }\end{array}$ & $\begin{array}{l}\text { Severe-moderate } \\
\text { anemia versus non- } \\
\text { anemic }\end{array}$ & $\begin{array}{l}\text { Mild anemia ver- } \\
\text { sus non-anemic }\end{array}$ & $\begin{array}{l}\text { Severe-moderate } \\
\text { anemia versus non- } \\
\text { anemic }\end{array}$ & $\begin{array}{l}\text { Mild anemia ver- } \\
\text { sus non-anemic }\end{array}$ \\
\hline No religion & $0.89(0.62,1.28)$ & $1.09(0.67,1.75)$ & $1.14(0.51,2.55)$ & $1.43(0.62,3.306)$ & $1.91(0.81,4.51)$ & $3.04(1.33,6.93)$ \\
\hline \multicolumn{7}{|l|}{ Ethnicity } \\
\hline Akan & Ref & Ref & Ref & Ref & Ref & Ref \\
\hline $\mathrm{Ga} /$ Dangme & $0.80(0.49,1.33)$ & $0.80(0.48,1.34)$ & $0.96(0.48,1.94)$ & $1.41(0.78,2.526)$ & $1.92(1.05,3.50)$ & $1.27(0.67,2.39)$ \\
\hline Ewe & $0.77(0.55,1.08)$ & $0.87(0.60,1.27)$ & $1.20(0.71,2.00)$ & $1.41(0.80,2.467)$ & $0.88(0.52,1.47)$ & $1.15(0.72,1.81)$ \\
\hline Mole-Dagbani & $0.88(0.58,1.33)$ & $1.20(0.78,1.86)$ & $0.95(0.55,1.62)$ & $1.45(0.79,2.656)$ & $0.62(0.34,1.12)$ & $0.95(0.53,1.70)$ \\
\hline Gurma & $0.90(0.51,1.58)$ & $0.93(0.50,1.75)$ & $2.05(1.04,4.05)$ & $1.73(0.75,3.971)$ & $1.10(0.42,2.86)$ & $1.50(0.65,3.46)$ \\
\hline Other & $0.77(0.50,1.18)$ & $0.96(0.60,1.55)$ & $0.93(0.52,1.66)$ & $1.36(0.72,2.565)$ & $0.87(0.47,1.60)$ & $1.30(0.70,2.40)$ \\
\hline
\end{tabular}

Bolded values are significant at the $<0.05$ level

Acknowledgements We are grateful to the interviewers and program staff who have conducted this survey.

Author Contributions LS conceived of the data analysis plan, completed the data analysis, and draft the manuscript. AJ contributed to the data analysis plan, helped interpret the data, and revised the manuscript for important intellectual content. MW contributed to the data analysis plan, helped interpret the data, and revised the manuscript for important intellectual content. All authors gave final approval of the manuscript to be published and agree to act as guarantors of the work.

Funding Bill and Melinda Gates Foundation (OPP1154099).

\section{Compliance with Ethical Standards}

Conflict of interests The authors have no conflicts of interest relevant to this article to disclose.

Open Access This article is licensed under a Creative Commons Attribution 4.0 International License, which permits use, sharing, adaptation, distribution and reproduction in any medium or format, as long as you give appropriate credit to the original author(s) and the source, provide a link to the Creative Commons licence, and indicate if changes were made. The images or other third party material in this article are included in the article's Creative Commons licence, unless indicated otherwise in a credit line to the material. If material is not included in the article's Creative Commons licence and your intended use is not permitted by statutory regulation or exceeds the permitted use, you will need to obtain permission directly from the copyright holder. To view a copy of this licence, visit http://creativecommons.org/licenses/by/4.0/.

\section{References}

Balarajan, Y., Ramakrishnan, U., Ozaltin, E., Shankar, A. H., \& Subramanian, S. V. (2011). Anaemia in low-income and middle-income countries. Lancet, 378, 2123-2135. https://doi.org/10.1016/S0140 $-6736(10) 62304-5$.

Beard, J. L., \& Connor, J. R. (2003). Iron status and neural functioning. Annual Review of Nutrition, 23(1), 41-58. https://doi.org/10.1146/ annurev.nutr.23.020102.075739.
Black, R. E., Victora, C. G., Walker, S. P., Bhutta, Z. A., Christian, P., de Onis, M., ... Maternal and Child Nutrition Study Group. (2013). Maternal and child undernutrition and overweight in low-income and middle-income countries. Lancet (London, England), 382(9890), 427-451. https://doi.org/10.1016/S0140 $-6736(13) 60937-X$.

Camaschella, C. (2015). Iron-deficiency anemia. New England Journal of Medicine, 372(19), 1832-1843. https://doi.org/10.1056/NEJMr a1401038.

Cameron, L., \& Williams, J. (2009). Is the relationship between socioeconomic status and health stronger for older children in developing countries? Demography, 46(2), 303-324. https://doi. org/10.1353/dem.0.0054.

Cumber, S. N., Bongkiynuy, N., Jaila, S., \& Tsoka-Gwegweni, J. M. (2017). Poor complementary feeding practices among young children in Cameroon. South African Journal of Clinical Nutrition, 30(2), 37-38.

Egbi, G., Steiner-Asiedu, M., Kwesi, F. S., Ayi, I., Ofosu, W., Setorglo, J.,... Armar-Klemesu, M. (2014). Anaemia among school children older than five years in the Volta Region of Ghana. The Pan African Medical Journal, 17(Suppl 1), 10. https://doi.org/10.11694/ pamj.supp.2014.17.1.3205.

Ewusie, J. E., Ahiadeke, C., Beyene, J., \& Hamid, J. S. (2014). Prevalence of anemia among under-5 children in the Ghanaian population: Estimates from the Ghana demographic and health survey. BMC Public Health, 14(1), 626. https://doi. org/10.1186/1471-2458-14-626.

Feinstein, J. S. (1993). The relationship between socioeconomic status and health: A review of the literature. The Milbank Quarterly, 71(2), 279-322.

Ghana Statistical Service. (2012). 2010 Population and housing census final results. Retrieved December 2017, from https://www.stats ghana.gov.gh/docfiles/2010phc/2010_POPULATION_AND_ HOUSING_CENSUS_FINAL_RESULTS.pdf.

Ghana Statistical Service, Ghana Health Service, \& ICF International. (2009). Ghana Demographic and Health Survey 2008. Retrieved August 13, 2012, from https://dhsprogram.com/pubs/pdf/FR221 /FR221.pdf.

Ghana Statistical Service, Ghana Health Service, \& ICF International. (2015). Ghana Demographic and Health Survey 2014. Retrieved December 2017, from www.DHSprogram.com.

Ghana Statistical Service, Noguchi Memorial Institute for Medical Research, \& ORC Macro. (2004). Ghana Demographic and 
Health Survey 2003. Retrieved December 2017, from https://dhspr ogram.com/pubs/pdf/FR152/FR152.pdf.

Habicht, J. P. (2000). The association between prolonged breastfeeding and poor growth-What are the implications? Advances in Experimental Medicine and Biology, 478, 193-200.

Klinkenberg, E., McCall, P. J., Wilson, M. D., Akoto, A. O., Amerasinghe, F. P., Bates, I.,... Donnelly, M. J. (2006). Urban malaria and anaemia in children: A cross-sectional survey in two cities of Ghana. Tropical Medicine and International Health, 11(5), 578-588. https://doi.org/10.1111/j.1365-3156.2006.01609.x.

Kotecha, P. V. (2011). Nutritional anemia in young children with focus on Asia and India. Indian Journal of Community Medicine: Official Publication of Indian Association of Preventive \& Social Medicine, 36(1), 8-16. https://doi.org/10.4103/0970-0218.80786.

Lander, R., Enkhjargal, T., Batjargal, J., Bolormaa, N., Enkhmyagmar, D., Tserendolgor, U.,... Gibson, R. (2009). Poor dietary quality of complementary foods is associated with multiple micronutrient deficiencies during early childhood in Mongolia. Public Health Nutrition, 13(9), 1304-1313. https://doi.org/10.1017/S136898000 9991856.

Larsen, D. A., Grisham, T., Slawsky, E., \& Narine, L. (2017). An individual-level meta-analysis assessing the impact of community-level sanitation access on child stunting, anemia, and diarrhea: Evidence from DHS and MICS surveys. PLOS Neglected Tropical Diseases, 11(6), e0005591. https://doi.org/10.1371/journ al.pntd.0005591.

Levy, A., Fraser, D., Rosen, S. D., Dagan, R., Deckelbaum, R. J., Coles, C., et al. (2005). Anemia as a risk factor for infectious diseases in infants and toddlers: Results from a prospective study. European Journal of Epidemiology, 20(3), 277-284. https://doi.org/10.1007/ s10654-004-6515-6.

McCann, J. C., \& Ames, B. N. (2007). An overview of evidence for a causal relationship between iron deficiency during development and deficits in cognitive or behavioral function. The American Journal of Clinical Nutrition, 85, 931-945.

Mueller, I., Rarau, P., Vaucher, P., Lombardo, P., Senn, N., \& Favrat, B. (2017). Hemoglobin levels and the risk of malaria in Papua New Guinean infants: A nested cohort study. The American Journal of Tropical Medicine and Hygiene. https://doi.org/10.4269/ajtmh .17-0093.

Neumann, C. G., Gewa, C., \& Bwibo, N. O. (2004). Child nutrition in developing countries. Pediatric Annals, 33(10), 658.

Ngnie-Teta, I., Kuate-Defo, B., \& Receveur, O. (2009). Multilevel modelling of sociodemographic predictors of various levels of anaemia among women in Mali. Public Health Nutrition, 12(9), 1462-1469. https://doi.org/10.1017/S1368980008004400.

Paganini, D., \& Zimmermann, M. B. (2017). Effects of iron fortification and supplementation on the gut microbiome and diarrhea in infants and children: A review. The American Journal of Clinical Nutrition. https://doi.org/10.3945/ajcn.117.156067.

Saaka, M., \& Galaa, S. Z. (2017). How is dietary diversity related to haematological status of preschool children in Ghana? Food \& Nutrition Research, 61(1), 1333389. https://doi. org/10.1080/16546628.2017.1333389.

Semba, R. D., de Pee, S., Ricks, M. O., Sari, M., \& Bloem, M. W. (2008). Diarrhea and fever as risk factors for anemia among children under age five living in urban slum areas of Indonesia. International Journal of Infectious Diseases, 12(1), 62-70. https://doi. org/10.1016/J.IJID.2007.04.011.
Siekmans, K., Receveur, O., \& Haddad, S. (2014). Can an integrated approach reduce child vulnerability to anaemia? Evidence from three African countries. PLOS ONE. https://doi.org/10.1371/journ al.pone. 0090108 .

Simondon, K. B., Costes, R., Delaunay, V., Diallo, A., \& Simondon, F. (2001). Children's height, health and appetite influence mothers' weaning decisions in rural Senegal. International Journal of Epidemiology, 30(3), 476-481.

Stevens, G. A., Finucane, M. M., De-Regil, L. M., Paciorek, C. J., Flaxman, S. R., Branca, F. ... Nutrition Impact Model Study Group (Anaemia). (2013). Global, regional, and national trends in haemoglobin concentration and prevalence of total and severe anaemia in children and pregnant and non-pregnant women for 19952011: A systematic analysis of population-representative data. The Lancet Global Health, 1(1), e16-25. https://doi.org/10.1016/ S2214-109X(13)70001-9.

United States Agency for International Development. (2017). The DHS Program. Retrieved July 27, 2017, from https://www.dhsprogram .com/.

VanBuskirk, K. M., Ofosu, A., Kennedy, A., \& Denno, D. M. (2014). Pediatric anemia in rural ghana: A cross-sectional study of prevalence and risk factors. Journal of Tropical Pediatrics, 60(4), 308-317. https://doi.org/10.1093/tropej/fmu020.

Villalpando, S., Shamah-Levy, T., Ramírez-Silva, C. I., MejíaRodríguez, F., \& Rivera, J. A. (2003). Prevalence of anemia in children 1 to 12 years of age. Results from a nationwide probabilistic survey in Mexico. Salud Publica de Mexico, 4(Suppl 4), S490-S498.

WHO. (2011). Haemoglobin concentrations for the diagnosis of anaemia and assessment of severity. Geneva: World Health Organization.

Woldie, H., Kebede, Y., \& Tariku, A. (2015). Factors associated with anemia among children aged 6-23 months attending growth monitoring at Tsitsika Health Center, Wag-Himra Zone, Northeast Ethiopia. Journal of Nutrition and Metabolism, 2015, 928632. https://doi.org/10.1155/2015/928632.

Working Group on Infant and Young Child Feeding Indicators. (2006). Developing and Validating Simple Indicators of Dietary Quality and Energy Intake of Infants and Young Children in Developing Countries: Summary of findings from analysis of 10 data sets. Retrieved December 2017, from www.fantaproject.org.

World Health Organization. (2008a). Indicators for assessing infant and young child feeding practices: Part 1 Definition. Retrieved from https://apps.who.int/iris/bitstream/handle/10665/43895 /9789241596664_eng.pdf?sequence=1.

World Health Organization. (2008b). The Global Burden of Disease: 2004 update. https://doi.org/10.1038/npp.2011.85.

World Health Organization. (2014). Preventing diarrhoea through better water, sanitation and hygiene. Geneva. Retrieved from https://apps.who.int/iris/bitstream/handle/10665/150112/97892 41564823_eng.pdf;jsessionid=5758DFB95F57DB9CF8362A5A6 FEBEDDA? sequence $=1$.

Publisher's Note Springer Nature remains neutral with regard to jurisdictional claims in published maps and institutional affiliations. 